\title{
Packings and Coverings of Various Complete Graphs with the 4-Cycle with a Pendant Edge
}

\author{
Brandon Coker, ${ }^{1}$ Gary D. Coker, ${ }^{2}$ Robert Gardner, ${ }^{3}$ and Yan Xia ${ }^{3}$ \\ ${ }^{1}$ Department of Computer Science, Francis Marion University, Florence, SC 29502, USA \\ ${ }^{2}$ Institute of Mathematics and Physics, Aberystwyth University, Aberystwyth, Ceredigion SY23 3BZ, UK \\ ${ }^{3}$ Department of Mathematics and Statistics, East Tennessee State University, Johnson City, TN 37614, USA
}

Correspondence should be addressed to Robert Gardner; gardnerr@etsu.edu

Received 17 June 2013; Accepted 12 August 2013

Academic Editors: C. da Fonseca, P. E. Jorgensen, A. V. Kelarev, and S. Zhou

Copyright (C) 2013 Brandon Coker et al. This is an open access article distributed under the Creative Commons Attribution License, which permits unrestricted use, distribution, and reproduction in any medium, provided the original work is properly cited.

We consider the packings and coverings of complete graphs with isomorphic copies of the 4-cycle with a pendant edge. Necessary and sufficient conditions are given for such structures for (1) complete graphs $K_{v}$, (2) complete bipartite graphs $K_{m, n}$, and (3) complete graphs with a hole $K(v, w)$. In the last two cases, we address both restricted and unrestricted coverings.

\section{Introduction, Motivation, and History}

A g-decomposition of graph $G$ is a set of subgraphs of $G, \gamma=$ $\left\{g_{1}, g_{2}, \ldots, g_{n}\right\}$, where $g_{i} \cong g$ for $i \in\{1,2, \ldots, n\}, E\left(g_{i}\right) \cap$ $E\left(g_{j}\right)=\varnothing$ for $i \neq j$, and $\cup_{i=1}^{n} E\left(g_{i}\right)=E(G)$. The $g_{i}$ are called blocks of the decomposition. The concept of a graph decomposition lies in the general area of the design theory. We can relate a graph decomposition to an experimental design by considering the following hypothetical situation: "suppose you have a collection of $v$ samples and you wish to compare a property of the samples. However, the only way to compare the samples is to run them three at a time in a machine which performs the comparison. The machine cannot be calibrated from run to run and so to compare two samples, we must run them together in the machine. When can all of the $v$ samples be optimally compared to each other by running the machine $\left(\begin{array}{c}v \\ 2\end{array}\right) / 3$ times?" The solution to this question is equivalent to finding a $K_{3}$-decomposition of $K_{v}$, where each vertex of $K_{v}$ represents a sample, an edge joining two vertices represents a comparison of the two corresponding samples, and a copy of $K_{3}$ represents a run of the machine. A $K_{3}$-decomposition of $K_{v}$ exists if and only if $v \equiv 1$ or $3(\bmod 6)$, and such a structure is called a Steiner triple system [1].

In the event that a $g$-decomposition of $G$ does not exist, we can still consider a set of isomorphic copies of graphs $g$ which "approximate" a decomposition. There are two approaches to this. We describe the two approaches in terms of the sample comparison analogy. In the first approach, we can try comparing as many of the samples as possible, without repetition of comparisons (it might be that running the machine is expensive). In the setting mentioned above, we could seek a collection of runs of the machine (represented by copies of $K_{3}$ ) which do not repeat pairs of samples run together (i.e., the copies of $K_{3}$ are edge disjoint), and which minimizes the number of pairs of samples which are omitted (i.e., the cardinality of the set of edges in $K_{v}$ which are in none of the copies of $K_{3}$ is made minimal). Such an experimental design is related to a maximal graph packing. A maximal $g$ packing of a graph $G$ with isomorphic copies of a graph $g$ is a set $\left\{g_{1}, g_{2}, \ldots, g_{n}\right\}$, where $g_{i} \cong g$ and $V\left(g_{i}\right) \subset V(G)$ for all $i$, $E\left(g_{i}\right) \cap E\left(g_{j}\right)=\varnothing$ for $i \neq j, \cup_{i=1}^{n} g_{i} \subset G$, and $\left|E(G) \backslash \cup_{i=1}^{n} E\left(g_{i}\right)\right|$ is minimal. In particular, the machine analogy corresponds to a $K_{3}$-packing of $K_{v}$. Such designs are explored in [2]. Other packings of the complete graphs have also been studied, for example, 4-cycle-packings [3], $K_{4}$-packings [4], and 6-cyclepackings $[5,6]$. A second approach involves comparing all of the samples to each other, but with minimal repetitions of the compared samples (we might postulate that the machine must have three samples in it during each run to keep it balanced). This experimental design is related to a minimal 


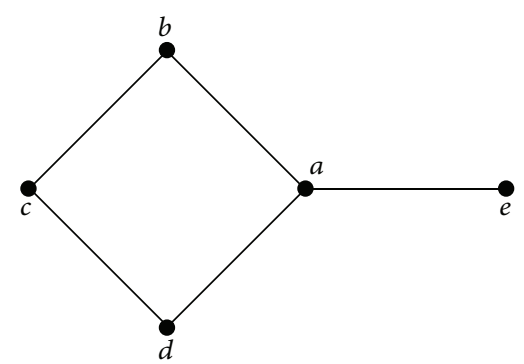

Figure 1: We denote by $H=[a, b, c, d ; e]$ this graph.

graph covering. A minimal $g$-covering of a graph $G$ with isomorphic copies of a graph $g$ is a set $\left\{g_{1}, g_{2}, \ldots, g_{n}\right\}$, where $g_{i} \cong g, V\left(g_{i}\right) \subset V(G), E\left(g_{i}\right) \subset E(G)$ for all $i, G \subset \cup_{i=1}^{n} g_{i}$, and $\left|\cup_{i=1}^{n} E\left(g_{i}\right) \backslash E(G)\right|$ is minimal (when considering coverings, the graph $\cup_{i=1}^{n} g_{i}$ may not be simple and $\cup_{i=1}^{n} E\left(g_{i}\right)$ may be a multiset). The machine analogy in this case corresponds to a $K_{3}$-covering of $K_{v}$. Such designs are explored in [7]. Coverings of $K_{v}$ have also been explored, for example, for 4cycles [2] and 6-cycles [8].

In terms of graph decompositions, several studies have concentrated on the $g$-decompositions of complete graphs into copies of a given graph $g$ with a small number of vertices [9-12]. In this paper, we go in a different direction and consider a single graph $g$, the 4 -cycle with a pendant edge, and explore packings and coverings of several graphs related to the complete graph. We denote the 4 -cycle with a pendant edge as $H=[a, b, c, d ; e]$, where $V(H)=\{a, b, c, d, e\}$ and $E(H)=\{(a, b),(b, c),(c, d),(a, d),(a, e)\}$. See Figure 1. An $H$ decomposition of $K_{v}$ exists if and only if $v \equiv 0$ or $1(\bmod 5)$, $v \geq 10$ [9]. An $H$-decomposition of the complete bipartite graph, $K_{m, n}$, exists if and only if $m n \equiv 0(\bmod 5), m \geq 5$, and $n \geq 2$ [13]. Another graph related to the complete graph is the complete graph with a hole $K(v, w)$. The complete graph on $v$ vertices with a hole of size $w$ is the graph with a vertex set $V(K(v, w))=V_{v-w} \cup V_{w}$, where $\left|V_{v-w}\right|=v-w$ and $\left|V_{w}\right|=w$, and edge set $E(K(v, w))=\{(a, b) \mid a, b \in$ $\left.V(K(v, w)),\{a, b\} \not \subset V_{w}\right\}$. Necessary and sufficient conditions for the decomposition of $K(v, w)$ into $m$-cycles are known for $m \in\{3,4,5,6,7,8,10,12,14\}[14-16]$. There is an $H$ decomposition of $K(v, w)$ if and only if $|E(K(v, w))| \equiv 0$ $(\bmod 5), v-w \geq 4$, and $(v, w) \notin\{(5,1),(6,1)\}[13]$.

The graph $K(v, w)$ relates to the experimental design story as follows. Suppose you have performed comparisons on a collection of $w$ samples and then received an additional collection of samples (say, $v-w$ new samples). You now wish to compare the $v-w$ new samples to each other and to the original $w$ samples. In the case of the machine described above, this would correspond to a $K_{3}$ decomposition of $K(v, w)$. In the event that a decomposition does not exist, we can explore the packings and coverings of $K(v, w)$. With a maximal $g$-packing of $G$, we require that each copy of $g$ is a subgraph of $G$. The definition given above for a maximal $g$-covering also involves the condition that each copy of $g$ is a subgraph of $G$. Most studies of coverings have involved $G=K_{v}$, so the condition that the copies of $g$ are subgraphs of $G$ is trivially satisfied. But when $G$ is not a complete graph, there is no obvious reason to impose the subgraph condition. Returning to the testing-of-samples story, we see no reason to disallow, for example, the testing (or retesting) of two samples in the hole of $K(v, w)$. Therefore, we are motivated to refine the definition of a graph covering into two cases-one case in which the edges that are not in $G$ are forbidden from use in the copies of $g$ and a second case in which these edges are not forbidden. A minimal unrestricted $g$-covering of a graph $G$ with isomorphic copies of a graph $g$ is a set $\left\{g_{1}, g_{2}, \ldots, g_{n}\right\}$ where $g_{i} \cong g, V\left(g_{i}\right) \subset V(G), G \subset \cup_{i=1}^{n} g_{i}$, and $\left|\cup_{i=1}^{n} E\left(g_{i}\right) \backslash G\right|$ is minimal (the graph $\cup_{i=1}^{n} g_{i}$ may not be simple and $\cup_{i=1}^{n} E\left(g_{i}\right)$ may be a multiset). A minimal restricted $g$-covering of a graph $G$ with isomorphic copies of a graph $g$ is a set $\left\{g_{1}, g_{2}, \ldots, g_{n}\right\}$, where $g_{i} \cong g, V\left(g_{i}\right) \subset V(G), E\left(g_{i}\right) \subset E(G)$ for all $i, G \subset$ $\cup_{i=1}^{n} g_{i}$, and $\left|\cup_{i=1}^{n} E\left(g_{i}\right) \backslash G\right|$ is minimal. The distinction between restricted and unrestricted coverings was introduced in [17]. Notice that in the event that $G$ is a complete graph, there is no distinction between a minimal restricted and minimal unrestricted covering.

The purpose of this paper is to give $H$-packings of $K_{v}$, $K_{m, n}$, and $K(v, w)$, as well as $H$-coverings of $K_{v}$, and restricted and unrestricted $H$-coverings of $K_{m, n}$ and $K(v, w)$.

\section{Packing and Covering $K_{v}$}

In this section, when necessary, we assume that the vertex set of $K_{v}$ is $V\left(K_{v}\right)=\{0,1,2, \ldots, v-1\}$. Since $H$ has 5 vertices, we only consider $v \geq 5$.

Theorem 1. A maximal $H$-packing of $K_{v}, v \geq 5$, has leave $L$, where $|E(L)|=\left|E\left(K_{v}\right)\right|(\bmod 5)$, except when $v \in\{5,6\}$ in which case $|E(L)|=5$.

Proof. Since $|E(H)|=5$, then it is necessary that in any $H$-packing of $K_{v}$ with leave $L,|E(L)| \equiv\left|E\left(K_{v}\right)\right|(\bmod 5)$. Therefore, such a packing with $|E(L)|=\left|E\left(K_{v}\right)\right|(\bmod 5)$ would be maximal. If $v \in\{5,6\}$, then $\left|E\left(K_{v}\right)\right| \equiv 0(\bmod 5)$, but there is not an $H$-decomposition of $K_{v}$ [9]. So for $v \in\{5,6\}$, an $H$-packing of $K_{v}$ with leave $L$, where $|E(L)|=5$ would be maximal.

Case 1. Suppose $v=5$. The set $\{[0,1,2,3 ; 4]\}$ is a maximal packing of $K_{5}$ with leave $L$, where $E(L)=\{(0,2),(1,3),(1,4)$, $(2,4),(3,4)\}$, so $|E(L)|=5$.

Case 2. Suppose $v=6$. The set $\{[0,1,2,3 ; 4],[1,3,4,5 ; 4]\}$ is a maximal packing of $K_{6}$ with leave $L$, where $E(L)=\{(0,2),(0$, 5), $(2,5),(2,4),(3,5)\}$, so $|E(L)|=5$.

Case 3. Suppose $v \equiv 2$ or $4(\bmod 5), v \geq 9$. Since $\left|E\left(K_{v}\right)\right| \equiv 1$ $(\bmod 5),|E(L)|=1$ would be optimal. Now $K(v, 2)$ can be decomposed [13], so $|E(L)|=1$.

Case 4. Suppose $v \equiv 3(\bmod 5), v \geq 8$. Since $\left|E\left(K_{v}\right)\right| \equiv 3$ $(\bmod 5),|E(L)|=3$ would be optimal. Now $K(v, 3)$ can be decomposed [13], so $|E(L)|=3$.

In the following result (and throughout this paper), we refer to an equality of the form $a=b(\bmod c)$. By this, we mean that $a \in\{0,1,2, \ldots, c-1\}$ and $a \equiv b(\bmod c)$. 
Theorem 2. A minimal $H$-covering of $K_{v}, v \geq 5$, has padding $P$, where $|E(P)|=-\left|E\left(K_{v}\right)\right|(\bmod 5)$, except when $v \in\{5,6\}$ in which case $|E(P)|=5$.

Proof. Since $|E(H)|=5$, then it is necessary that in any $H$ covering of $K_{v}$ with padding $P$, we have $\left|E\left(K_{n}\right)\right|+|E(P)| \equiv 0$ $(\bmod 5)$ or that $|E(P)| \equiv-\left|E\left(K_{v}\right)\right|(\bmod 5)$. So if $|E(P)|=$ $-\left|E\left(K_{v}\right)\right|(\bmod 5)$, then the covering is minimal. If $v \in\{5,6\}$, then $-\left|E\left(K_{v}\right)\right| \equiv 0(\bmod 5)$, but there is no $H$-decomposition of $K_{v}$ [9]. So for $v \in\{5,6\}$, an $H$-covering of $K_{v}$ with padding $P$, where $|E(P)|=5$ would be minimal.

Case 1. Suppose $v=5$. The set $\{[0,1,2,3 ; 4],[1,2,0,4 ; 3],[4,1$, $0,2 ; 3]\}$ is a minimal covering of $K_{5}$ with padding $P$, where $(P)=\{(0,1),(0,2),(0,4),(1,2),(1,4)\}$, so $|E(P)|=5$.

Case 2. Suppose $v=6$. The set $\{[0,1,2,3 ; 4],[5,0,2,4 ; 1],[5$, $3,4,1 ; 2],[3,4,5,2 ; 1]\}$ is a minimal covering of $K_{6}$ with padding $P$, where $E(P)=\{(1,5),(2,3),(2,5),(3,4),(4,5)\}$, so $|E(P)|=5$.

Case 3. Suppose $v \equiv 2$ or $4(\bmod 5), v \geq 7$. There is an $H$ decomposition of $K(v, 2)$ [13]. Take such a decomposition, along with another copy of $H$ which includes the edge of the hole of $K(v, 2)$. This gives a covering of $K_{v}$ with padding $P$, where $|E(P)|=4=-\left|E\left(K_{v}\right)\right|(\bmod 5)$.

Case 4. Suppose $v \equiv 3(\bmod 5), v \geq 8$. An $H$-covering of $K_{8}$ is given by $\{[0,1,2,7 ; 3],[1,3,5,7 ; 6],[4,5,6,3 ; 7],[2,4,6$, $0 ; 5],[1,4,0,5 ; 2],[7,3,2,6 ; 0]\}$ with padding $P$, where $E(P)=$ $\{(0,7),(1,2)\}$ and the covering is optimal. For $v \geq 13, K_{v}=$ $K(v, 8) \cup K_{8}, K(v, 8)$ can be decomposed [13], and $K_{8}$ can be covered with padding $P$, where $|E(P)|=2$. Therefore, there is an optimal $H$-covering of $K_{v}$ with padding $P$, where $|E(P)|=$ $2=-\left|E\left(K_{v}\right)\right|(\bmod 5)$.

\section{Packing and Covering the Complete Bipartite Graph}

In this section, we consider the $H$-packings and $H$-coverings of the complete bipartite graph $K_{m, n}$. We assume the partite sets of $K_{m, n}$ are $\left\{0_{0}, 1_{0}, \ldots,(m-1)_{0}\right\}$ and $\left\{0_{1}, 1_{1}, \ldots,(n-1)_{1}\right\}$.

Theorem 3. A maximal $H$-packing of $K_{m, n}$ has leave $L$, where

(1) $|E(L)|=m n$ if $m=1$ or $n=1$, or if $m=n=2$, or

(2) $|E(L)|=\left|E\left(K_{m, n}\right)\right|(\bmod 5)$, otherwise.

Proof. First, if $m$ or $n$ equals 1 , then $H$ is not a subgraph of $K_{m, n}$, and the leave must have $m n$ edges. Similarly, the leave of a packing of $K_{2,2}$ has $m n=4$ edges. For $m \geq 2$ and $n \geq 3$, as in the proof of Theorem 1, an $H$-packing of $K_{m, n}$ with leave $L$, where $|E(L)|=\left|E\left(K_{m, n}\right)\right|(\bmod 5)$ would be maximal. Next, for $m \geq 2$ and $n \geq 3$ we observe that if there is a packing of $K_{m, n}$ with leave $L$, then there is a packing of $K_{m+5 i, n+5 j}$ with leave $L$ for all $i, j \in \mathbb{N}$. This is because $K_{m+5 i, n+5 j}=K_{m, n} \cup K_{m, 5 j} \cup K_{5 i, n} \cup K_{5 i, 5 j}$, where the partite sets of $K_{m+5 i, n+5 j}$ are $\left\{0_{0}, 1_{0}, \ldots,(m-1+5 i)_{0}\right\}$ and $\left\{0_{1}, 1_{1}, \ldots,(n-1+5 j)_{1}\right\}$, the partite sets of $K_{m, n}$ are $\left\{0_{0}, 1_{0}, \ldots,(m-1)_{0}\right\}$ and $\left\{0_{1}, 1_{1}, \ldots,(n-1)_{1}\right\}$, the partite sets of $K_{m, 5 j}$ are $\left\{0_{0}, 1_{0}, \ldots,(m-1)_{0}\right\}$ and $\left\{n_{1},(n+1)_{1}, \ldots,(n-1+\right.$ $\left.5 j)_{1}\right\}$, the partite sets of $K_{5 i, n}$ are $\left\{m_{0},(m+1)_{0}, \ldots,(m-1+5 i)_{0}\right\}$ and $\left\{0_{1}, 1_{1}, \ldots,(n-1)_{1}\right\}$, and the partite sets of $K_{5 i, 5 j}$ are $\left\{m_{0},(m+1)_{0}, \ldots,(m-1+5 i)_{0}\right\}$ and $\left\{n_{1},(n+1)_{1}, \ldots,(n-\right.$ $\left.1+5 j)_{1}\right\}$. There is an $H$-decomposition of $K_{m, 5 j}, K_{5 i, n}$, and $K_{5 i, 5 j}[13]$.

In Table 1, the packings, combined with the decompositions of complete bipartite graphs mentioned above, yield the result.

Theorem 4. A minimal restricted $H$-covering of $K_{m, n}$, where neither $m$ nor $n$ equals 1 and $m+n \geq 5$, has padding $P$, where $|E(P)|=-\left|E\left(K_{m, n}\right)\right|(\bmod 5)$.

Proof. For $K_{1, n}, H$ is not a subgraph and so a restricted $H$-covering does not exist. Similar to the argument in Theorem 2, a $H$-covering of $K_{m, n}$ with padding $P$ where $|E(P)|=-\left|E\left(K_{m, n}\right)\right|(\bmod 5)$ would be minimal. As in Theorem 3 , for $m \geq 2$ and $m \geq 3$, if there is a restricted covering of $K_{m, n}$ with padding $P$, then there is a restricted covering of $K_{m+5 i, n+5 j}$ with padding $P$ for all $i, j \in \mathbb{N}$.

In Table 2, the coverings, combined with the decompositions of complete graphs mentioned in Theorem 3, yield the result.

Theorem 5. A minimal unrestricted $H$-covering of $K_{m, n}$ has padding $P$ where

(1) when $m>1$ and $n>1,|E(P)|=-\left|E\left(K_{m, n}\right)\right|(\bmod 5)$,

(2) when $m=1,|E(P)|=(2 / 3) n$ for $n \equiv 0(\bmod 3)$, $|E(P)|=2(n+5) / 3$ for $n \equiv 1(\bmod 3),|E(P)|=(2 n+$ $5) / 3$ for $n \equiv 2(\bmod 3)$.

Proof. For $m>1$ and $n>1$, the necessary condition follows as in the proof of Theorem 4 . In this case, sufficiency also follows from Theorem 4 .

When $m=1$, a copy of $H$ where $V(H) \subset V\left(K_{1, n}\right)$ has at most 3 edges in $E\left(K_{1, n}\right)$ and at least 2 edges in the padding. So in an $H$-covering of $K_{1, n}$, there are at least $\lceil n / 3\rceil$ copies of $H$. Now $\lfloor n / 3\rfloor$ copies of $H$ can have at most $3\lfloor n / 3\rfloor$ edges in $E\left(K_{1, n}\right)$ and at least $2\lfloor n / 3\rfloor$ edges in the padding. If $n \equiv 1$ $(\bmod 3)$, then to completely cover $K_{1, n}$ we must add one more copy of $H$ which has at most 1 edge in $E\left(K_{1, n}\right)$ and at least 4 edges in the padding. If $n \equiv 2(\bmod 3)$, then to completely cover $K_{1, n}$ we must add one more copy of $H$ which has at most 2 edges in $E\left(K_{1, n}\right)$ and at least 3 edges in the padding. This yields the necessary conditions for $m=1$. We now establish sufficiency for $m=1$.

Case 1. Suppose $m=1$ and $n \equiv 0(\bmod 3) ; n \geq 6$. Consider the blocks $\left\{\left[0_{0}, 0_{1}, 3_{1}, 1_{1} ; 2_{1}\right]\right\} \cup\left\{\left[0_{0},(3 k)_{1}, 2_{1},(3 k+1)_{1} ;(3 k+\right.\right.$ $\left.\left.2)_{1}\right] \mid k=1,2, \ldots,(n / 3)-1\right\}$. This is a covering of $K_{m, n}$ with padding $P=\left\{\left(0_{1}, 3_{1}\right),\left(1_{1}, 3_{1}\right)\right\} \cup\left\{\left(2_{1},(3 k)_{1}\right),\left(2_{1},(3 k+1)_{1}\right) \mid\right.$ $k=1,2, \ldots,(n / 3)-1\}$, where $|E(P)|=(2 / 3) n$.

Case 2. Suppose $m=1$ and $n \equiv 1(\bmod 3) ; n \geq 4$. From Case 1 , there is a covering of $K_{1, n-1}$, where the partite sets of $K_{1, n-1}$ 
TABLE 1

\begin{tabular}{|c|c|c|c|}
\hline$(m, n)(\bmod 5)$ & $K_{m, n}$ & Packing & Leave \\
\hline$(1,1)$ & $K_{6,6}$ & $\begin{array}{c}\left\{\left[0_{0}, 0_{1}, 1_{0}, 1_{1} ; 2_{1}\right],\left[0_{0}, 5_{1}, 1_{0}, 4_{1} ; 3_{1}\right],\left[2_{0}, 0_{1}, 3_{0}, 1_{1} ; 2_{1}\right]\right. \\
{\left[2_{0}, 4_{1}, 3_{0}, 5_{1} ; 3_{1}\right],\left[4_{0}, 0_{1}, 5_{0}, 1_{1} ; 2_{1}\right],\left[4_{0}, 4_{1}, 5_{0}, 5_{1} ; 3_{1}\right]} \\
\left.\left[2_{1}, 1_{0}, 3_{1}, 3_{0} ; 5_{0}\right]\right\}\end{array}$ & $\left\{\left(5_{0}, 3_{1}\right)\right\}$ \\
\hline$(1,2)$ & $K_{6,2}$ & $\left\{\left[0_{1}, 0_{0}, 1_{1}, 1_{0} ; 2_{0}\right],\left[1_{1}, 5_{0}, 0_{1}, 4_{0} ; 3_{0}\right]\right\}$ & $\left\{\left(2_{0}, 1_{1}\right),\left(3_{0}, 0_{1}\right)\right\}$ \\
\hline$(1,3)$ & $K_{6,3}$ & $\left\{\left[3_{0}, 0_{1}, 2_{0}, 1_{1} ; 2_{1}\right],\left[1_{0}, 0_{1}, 0_{0}, 1_{1} ; 2_{1}\right],\left[5_{0}, 0_{1}, 4_{0}, 1_{1} ; 2_{1}\right]\right\}$ & $\left\{\left(0_{0}, 2_{1}\right),\left(2_{0}, 2_{1}\right),\left(4_{0}, 2_{1}\right)\right\}$ \\
\hline$(1,4)$ & $K_{6,4}$ & $\begin{array}{r}\left\{\left[0_{0}, 0_{1}, 1_{0}, 1_{1} ; 2_{1}\right],\left[3_{1}, 2_{0}, 2_{1}, 3_{0} ; 1_{0}\right],\right. \\
\left.\left[2_{1}, 4_{0}, 1_{1}, 5_{0} ; 1_{0}\right],\left[3_{1}, 4_{0}, 0_{1}, 5_{0} ; 0_{0}\right]\right\}\end{array}$ & $\begin{array}{r}\left\{\left(2_{0}, 0_{1}\right),\left(2_{0}, 1_{1}\right)\right. \\
\left.\quad\left(3_{0}, 0_{1}\right),\left(3_{0}, 1_{1}\right)\right\}\end{array}$ \\
\hline$(2,2)$ & $K_{2,2}$ & $\varnothing$ & $\begin{array}{l}\left\{\left(0_{0}, 0_{1}\right),\left(0_{0}, 1_{1}\right)\right. \\
\left.\left(1_{0}, 0_{1}\right),\left(1_{0}, 1_{1}\right)\right\}\end{array}$ \\
\hline$(2,3)$ & $K_{3,2}$ & $\left\{\left[1_{1}, 1_{0}, 0_{1}, 0_{0} ; 2_{0}\right]\right\}$ & $\left\{\left(2_{0}, 0_{1}\right)\right\}$ \\
\hline$(2,4)$ & $K_{4,2}$ & $\left\{\left[1_{1}, 1_{0}, 0_{1}, 0_{0} ; 2_{0}\right]\right\}$ & $\left\{\left(2_{0}, 0_{1}\right),\left(3_{0}, 0_{1}\right),\left(3_{0}, 1_{1}\right)\right\}$ \\
\hline$(3,3)$ & $K_{3,3}$ & $\left\{\left[1_{1}, 1_{0}, 0_{1}, 0_{0} ; 2_{0}\right]\right\}$ & $\begin{array}{r}\left\{\left(0_{0}, 2_{1}\right),\left(1_{0}, 2_{1}\right)\right. \\
\left.\left(2_{0}, 0_{1}\right),\left(2_{0}, 2_{1}\right)\right\}\end{array}$ \\
\hline$(3,4)$ & $K_{4,3}$ & $\left\{\left[0_{0}, 0_{1}, 1_{0}, 1_{1} ; 2_{1}\right],\left[2_{0}, 3_{1}, 1_{0}, 2_{1} ; 1_{1}\right]\right\}$ & $\left\{\left(0_{0}, 3_{1}\right),\left(2_{0}, 0_{1}\right)\right\}$ \\
\hline$(4,4)$ & $K_{4,4}$ & $\left\{\left[1_{0}, 1_{1}, 0_{0}, 0_{1} ; 2_{1}\right],\left[3_{0}, 1_{1}, 2_{0}, 0_{1} ; 2_{1}\right],\left[3_{1}, 2_{0}, 2_{1}, 0_{0} ; 3_{0}\right]\right\}$ & $\left\{\left(1_{0}, 3_{1}\right)\right\}$ \\
\hline
\end{tabular}

TABLE 2

\begin{tabular}{lccc}
\hline$(m, n)(\bmod 5)$ & $K_{m, n}$ & Covering & Padding \\
\hline$(1,1)$ & $K_{6,6}$ & $\left\{\left[3_{1}, 0_{0}, 0_{1}, 1_{0} ; 5_{0}\right]\right\}$ & $\left\{\left(0_{0}, 3_{1}\right),\left(0_{0}, 0_{1}\right),\left(1_{0}, 0_{1}\right),\left(1_{0}, 3_{1}\right)\right\}$ \\
$(1,2)$ & $K_{6,2}$ & $\left.\left\{0_{1}, 2_{0}, 1_{1}, 1_{0} ; 3_{0}\right]\right\}$ & $\left\{\left(1_{0}, 0_{1}\right),\left(1_{0}, 1_{1}\right),\left(2_{0}, 0_{1}\right)\right\}$ \\
$(1,3)$ & $K_{6,3}$ & $\left\{\left[2_{1}, 0_{0}, 1_{1}, 2_{0} ; 4_{0}\right]\right\}$ & $\left\{\left(0_{0}, 1_{1}\right),\left(2_{0}, 1_{1}\right)\right\}$ \\
$(1,4)$ & $K_{6,4}$ & $\left.\left\{2_{0}, 0_{1}, 3_{0}, 1_{1} ; 2_{1}\right]\right\}$ & $\left\{\left(2_{0}, 2_{1}\right)\right\}$ \\
$(2,2)$ & $K_{7,2}$ & $\left\{\left[0_{1}, 0_{0}, 1_{1}, 1_{0} ; 6_{0}\right],\left[1_{1}, 2_{0}, 0_{1}, 3_{0} ; 6_{0}\right],\left[1_{1}, 5_{0}, 0_{1}, 4_{0} ; 0_{0}\right]\right\}$ & $\left\{\left(0_{0}, 1_{1}\right)\right\}$ \\
$(2,3)$ & $K_{3,2}$ & $\left\{\left[0_{1}, 1_{0}, 1_{1}, 0_{0} ; 2_{0}\right]\right\}$ & $\left\{\left(0_{0}, 0_{1}\right),\left(0_{0}, 1_{1}\right),\left(1_{0}, 0_{1}\right),\left(1_{0}, 1_{1}\right)\right\}$ \\
$(2,4)$ & $K_{4,2}$ & $\left\{\left[0_{1}, 3_{0}, 1_{1}, 0_{0} ; 2_{0}\right]\right\}$ & $\left\{\left(0_{0}, 0_{1}\right),\left(0_{0}, 1_{1}\right)\right\}$ \\
$(3,3)$ & $K_{3,3}$ & $\left\{\left[2_{1}, 2_{0}, 0_{1}, 1_{0} ; 0_{0}\right]\right\}$ & $\left\{\left(1_{0}, 0_{1}\right)\right\}$ \\
$(3,4)$ & $K_{4,3}$ & $\left\{\left[0_{1}, 0_{0}, 3_{1}, 2_{0} ; 1_{0}\right]\right\}$ & $\left\{\left(0_{0}, 0_{1}\right),\left(1_{0}, 0_{1}\right),\left(2_{0}, 3_{1}\right)\right\}$ \\
$(4,4)$ & $K_{4,4}$ & $\left\{\left[3_{1}, 0_{0}, 0_{1}, 3_{0} ; 1_{0}\right]\right\}$ & $\left\{\left(0_{0}, 3_{1}\right),\left(0_{0}, 0_{1}\right),\left(3_{0}, 0_{1}\right),\left(3_{0}, 3_{1}\right)\right\}$ \\
\hline
\end{tabular}

are $\left\{0_{0}\right\}$, and $V_{n} \backslash\left\{(n-1)_{1}\right\}$ with padding $P_{1}$, where $\left|E\left(P_{1}\right)\right|=$ $2(n-1) / 3$. This covering along with $\left\{\left[0_{0}, 0_{1}, 2_{1}, 1_{1} ; 3_{1}\right]\right\}$, is an unrestricted covering of $K_{m, n}$ with padding $P_{2}=P_{1} \cup$ $\left\{\left(0_{0}, 0_{1}\right),\left(0_{1}, 2_{1}\right),\left(2_{1}, 1_{1}\right),\left(0_{0}, 1_{1}\right)\right\}$ and so $\left|E\left(P_{2}\right)\right|=2(n+5) / 3$.

Case 3. Suppose $m=1$ and $n \equiv 2(\bmod 3) ; n \geq 5$. From Case 1 , there is a covering of $K_{1, n-2}$, where the partite sets of $K_{1, n-2}$ are $\left\{0_{0}\right\}$ and $V_{n} \backslash\left\{(n-2)_{1}\right\}$ with padding $P_{1}$, where $\left|E\left(P_{1}\right)\right|=2(n-$ $2) / 3$. This covering along with $\left\{\left[00_{0},(n-1)_{1}, 0_{1},(n-2)_{1} ; 1_{1}\right]\right\}$ is an unrestricted covering of $K_{m, n}$ with padding $P_{2}=P_{1} \cup$ $\left\{\left(0_{1},(n-1)_{1}\right),\left(0_{1},(n-2)_{1}\right),\left(0_{0}, 1_{1}\right)\right\}$, and so $\left|E\left(P_{2}\right)\right|=(2 n+$ $5) / 3$.

\section{Packing the Complete Graph with a Hole}

In this section, we assume the vertex set of $K(v, w)$ is $V(K(v, w))=V_{v-w} \cup V_{w}$ as described in Section 1, where $V_{v-w}=\left\{0_{0}, 1_{0}, \ldots,(v-w-1)_{0}\right\}$ and $V_{w}=\left\{0_{1}, 1_{1}, \ldots,(w-1)_{1}\right\}$.

Theorem 6. A maximal $H$-packing of $K(v, w)$ has leave $L$, where $|E(L)|=|E(K(v, w))|(\bmod 5)$ and $v-w \geq 2$ is necessary.
Proof. When $v=w+1, H$ is not a subgraph of $K(v, w)$, and so, there is no packing. Therefore, $v-w \geq 2$ is necessary for the existence of a packing.

Case 1. If $v-w=6$, then $K(v, w)=K_{6} \cup K_{6, w}$, where the vertex set of $K_{6}$ is $V_{v-w}$ and the partite sets of $K_{6, w}$ are $V_{v-w}$ and $V_{w}$. There exists a packing $K_{6, w}$ with leave $L_{2}$ such that $\left|E\left(L_{2}\right)\right| \in\{1,2,3,4\}$. Without loss of generality, $\left(0_{0}, 0_{1}\right) \in E\left(L_{2}\right)$. Take such a packing along with $\left\{\left[4_{0}, 5_{0}\right.\right.$, $\left.\left.2_{0}, 3_{0} ; 1_{0}\right],\left[0_{0}, 3_{0}, 1_{0}, 5_{0} ; 2_{0}\right],\left[0_{0}, 1_{0}, 2_{0}, 4_{0} ; 0_{1}\right]\right\}$. This yields a packing of $K(v, w)$ with leave $L=\left\{\left(3_{0}, 5_{0}\right)\right\} \cup E\left(L_{2}\right) \backslash\left\{\left(0_{0}, 0_{1}\right)\right\}$, so $|E(L)|=\left|E\left(L_{2}\right)\right|$.

Case 2. Suppose $v \equiv 0(\bmod 5)$ and $w \equiv 2(\bmod 5)$. Then $K(v, w)=K_{v-w} \cup K_{v-w, w}$, where $V\left(K_{v-w}\right)=V_{v-w}$ and the partite sets of $K_{v-w, w}$ are $V_{v-w}$ and $V_{w}$. We have $v-w \equiv 3$ $(\bmod 5)$ and $w \equiv 2(\bmod 5)$. There is a maximal packing of $K_{v-w}$, where $v-w \equiv 3(\bmod 5)$ with $\left|E\left(L_{1}\right)\right|=3$ by Theorem 1 and a maximal packing of $K_{v-w, w}$ with $\left|E\left(L_{2}\right)\right|=1$ by Theorem 3. Therefore, there is a maximal packing of $K(v, w)$ with leave $L$, where $|E(L)|=4=|E(K(v, w))|(\bmod 5)$.

Case 3. Suppose $v \equiv 0(\bmod 5)$ and $w \equiv 3(\bmod 5)$ or $v \equiv 3$ $(\bmod 5)$ and $w \equiv 4(\bmod 5)$. Then $K(v, w)=K_{v-w} \cup K_{v-w, w}$ 
as in Case 2 , where $v-w \equiv 2(\bmod 5)$ and $w \equiv 3(\bmod 5)$ or $v-w \equiv 4(\bmod 5)$ and $w \equiv 4(\bmod 5)$. There is a maximal packing of $K_{v-w}$, where $v-w \equiv 2(\bmod 5)$ or $v-w \equiv 4(\bmod 5)$ with $\left|E\left(L_{1}\right)\right|=1$ by Theorem 1 , and there is a maximal packing of $K_{v-w, w}$ with $\left|E\left(L_{2}\right)\right|=1$ by Theorem 3 . Therefore, there is a maximal packing of $K(v, w)$ with leave $L$, where $|E(L)|=2=|E(K(v, w))|(\bmod 5)$.

Case 4. Suppose $v \equiv 0(\bmod 5)$ and $w \equiv 4(\bmod 5)$. When $v-w \geq 11, K(v, w)=K_{v-w} \cup K_{v-w, w}$, as in Case 2, where $v-w \equiv 1(\bmod 5)$ and $w \equiv 4(\bmod 5)$. There is a maximal packing of $K_{v-w, w}$ with $\left|E\left(L_{2}\right)\right|=4$ by Theorem 3 and $K_{v-w}$, where $v-w \equiv 1(\bmod 5)$ is decomposable [9]. Therefore, there is a maximal packing of $K(v, w)$ with leave $L$, where $|E(L)|=$ $4=|E(K(v, w))|(\bmod 5)$.

Case 5. Suppose $v \equiv 1(\bmod 5)$ and $w \equiv 2(\bmod 5)$ or $v \equiv 1$ $(\bmod 5)$ and $w \equiv 4(\bmod 5)$. Then $K(v, w)=K_{v-w} \cup K_{v-w, w}$, as in Case 2 , where $v-w \equiv 4(\bmod 5)$ and $w \equiv 2(\bmod 5)$, or $v-w \equiv 2(\bmod 5)$ and $w \equiv 4(\bmod 5)$. There is a maximal packing of $K_{v-w}$, where $v-w \equiv 4(\bmod 5)$ or $v-w \equiv 2(\bmod 5)$ with $\left|E\left(L_{1}\right)\right|=1$ by Theorem 1 , and there is a maximal packing of $K_{v-w, w}$ with $\left|E\left(L_{2}\right)\right|=3$ by Theorem 3 . Therefore, there is a maximal packing of $K(v, w)$ with leave $L$, where $|E(L)|=$ $4=|E(K(v, w))|(\bmod 5)$.

Case 6. Suppose $v \equiv 1(\bmod 5)$ and $w \equiv 3(\bmod 5)$. Then $K(v, w)=K_{v-w+1} \cup K_{v-w, w-1}$, where $V\left(K_{v-w+1}\right)=V_{v-w} \cup\left\{w_{1}\right\}$ and the partite sets of $K_{v-w, w-1}$ are $V_{v-w} \cup\left\{w_{1}\right\}$ and $V_{w} \backslash\left\{w_{1}\right\}$. Then there is a maximal packing of $K_{v-w+1}$ with leave $L_{1}$, where $\left|E\left(L_{1}\right)\right|=1$ by Theorem 1 , and there is a maximal packing of $K_{v-w, w-1}$ with leave $L_{2}$, where $\left|E\left(L_{2}\right)\right|=1$ by Theorem 3. Therefore, there is a maximal packing of $K(v, w)$ with leave $L$, where $|E(L)|=2=|E(K(v, w))|(\bmod 5)$.

Case 7. Suppose $v \equiv 2(\bmod 5)$ and $w \equiv 0(\bmod 5)$ or $v \equiv 4$ $(\bmod 5)$, and $w \equiv 0(\bmod 5)$. Then $K(v, w)=K_{v-w} \cup K_{v-w, w}$ where $v-w \equiv 2(\bmod 5)$ and $w \equiv 0(\bmod 5)$ or $v-w \equiv 4$ $(\bmod 5)$, and $w \equiv 0(\bmod 5)$. There is a maximal packing of $K_{v-w}$, where $v-w \equiv 2(\bmod 5)$ or $v-w \equiv 4(\bmod 5)$ with $\left|E\left(L_{1}\right)\right|=1$ by Theorem 1 and $K_{v-w, w}$ is decomposable [13]. Therefore, there is a maximal packing of $K(v, w)$ with leave $L$ where $|E(L)|=1=|E(K(v, w))|(\bmod 5)$.

Case 8. Suppose $v \equiv 2(\bmod 5)$ and $w \equiv 1(\bmod 5)$. Similar to Case 3, when $v-w \geq 11, K(v, w)=K_{v-w} \cup K_{v-w, w}$, as in Case 2 , where $v-w \equiv 1(\bmod 5)$ and $w \equiv 1(\bmod 5)$. There is a maximal packing of $K_{v-w, w}$ with $\left|E\left(L_{2}\right)\right|=1$ by Theorem 3 and $K_{v-w}$, where $v-w \equiv 1(\bmod 5)$ is decomposable [9]. Therefore, there is a maximal packing of $K(v, w)$ with leave $L$, where $|E(L)|=1=|E(K(v, w))|(\bmod 5)$.

Case 9. Suppose $v \equiv 2(\bmod 5)$ and $w \equiv 3(\bmod 5)$, or $v \equiv 3$ $(\bmod 5)$ and $w \equiv 1(\bmod 5)$. Then $K(v, w)=K_{v-w} \cup K_{v-w, w}$, as in Case 2 , where $v-w \equiv 4(\bmod 5), w \equiv 3(\bmod 5)$ or $v-w \equiv 2$ $(\bmod 5)$ and $w \equiv 1(\bmod 5)$. There is a maximal packing of $K_{v-w}$, where $v-w \equiv 4(\bmod 5)$ or $v-w \equiv 2(\bmod 5)$ with $\left|E\left(L_{1}\right)\right|=1$ by Theorem 1 , and there is a maximal packing of $K_{v-w, w}$ with $\left|E\left(L_{2}\right)\right|=2$ by Theorem 3 . Therefore, there is a maximal packing of $K(v, w)$ with leave $L$, where $|E(L)|=3=$ $|E(K(v, w))|(\bmod 5)$.

Case 10. Suppose $v \equiv 3(\bmod 5)$ and $w \equiv 0(\bmod 5)$. Then $K(v, w)=K_{v-w} \cup K_{v-w, w}$, as in Case 2, where $v-w \equiv 3$ $(\bmod 5)$ and $w \equiv 0(\bmod 5)$. There is a maximal packing of $K_{v-w}$, where $v-w \equiv 3(\bmod 5)$ with $\left|E\left(L_{1}\right)\right|=3$ by Theorem 1 and $K_{v-w, w}$ is decomposable [13]. Therefore, there is a maximal packing of $K(v, w)$ with leave $L$, where $|E(L)|=$ $3=|E(K(v, w))|(\bmod 5)$.

Case 11. Suppose $v \equiv 3(\bmod 5)$ and $w \equiv 2(\bmod 5)$. Similar to Case 4, when $v-w \geq 11, K(v, w)=K_{v-w} \cup K_{v-w, w}$, where $v-w \equiv 1(\bmod 5)$ and $w \equiv 2(\bmod 5)$. There is a maximal packing of $K_{v-w, w}$ with $\left|E\left(L_{2}\right)\right|=2$ by Theorem 3 and $K_{v-w}$ where $v-w \equiv 1(\bmod 5)$ is decomposable [9]. Therefore, there is a maximal packing of $K(v, w)$ with leave $L$, where $|E(L)|=$ $2=|E(K(v, w))|(\bmod 5)$.

Case 12. Suppose $v \equiv 4(\bmod 5)$ and $w \equiv 1(\bmod 5)$. As in Case 6, we have $K(v, w)=K_{v-w+1} \cup K_{v-w, w-1}$. Then there is a maximal packing of $K_{v-w+1}$ with leave $L_{1}$, where $\left|E\left(L_{1}\right)\right|=1$ by Theorem 1 and $K_{v-w, w-1}$ is decomposable [13]. There is a maximal packing of $K(v, w)$ with leave $L$, where $|E(L)|=1=$ $|E(K(v, w))|(\bmod 5)$.

Case 13. Suppose $v \equiv 4(\bmod 5)$ and $w \equiv 3(\bmod 5)$. Similar to Case 4, when $v-w \geq 11$, consider $K(v, w)=K_{v-w} \cup K_{v-w, w}$, where $v-w \equiv 1(\bmod 5)$ and $w \equiv 3(\bmod 5)$. There is a maximal packing of $K_{v-w, w}$ with $\left|E\left(L_{2}\right)\right|=3$ by Theorem 3 and $K_{v-w}$, where $v-w \equiv 1(\bmod 5)$ is decomposable [9]. Therefore, there is a maximal packing of $K(v, w)$ with leave $L$, where $|E(L)|=3=|E(K(v, w))|(\bmod 5)$.

\section{Covering the Complete Graph with a Hole}

As in the previous section, we assume the vertex set of $K(v, w)$ is $V(K(v, w))=V_{v-w} \cup V_{w}$, where $V_{v-w}=\left\{0_{0}, 1_{0}, \ldots,(v-w-\right.$ $\left.1)_{0}\right\}$ and $V_{w}=\left\{0_{1}, 1_{1}, \ldots,(w-1)_{1}\right\}$.

Theorem 7. A minimal restricted $H$-covering of $K(v, w)$ has padding $P$, where $|E(P)|=-|E(K(v, w))|(\bmod 5)$, when $v-$ $w>2$. No restricted $H$-covering of $K(v, w)$ exists for $v-w=2$.

Proof. First, suppose $v-w=2$. Consider the edge $\left(0_{0}, 1_{0}\right)$. If $\left(0_{0}, 1_{0}\right)$ is the pendant edge of an $H$, say $H=\left[0_{0}, a, b, c ; 1_{0}\right]$, then $0_{0}, 1_{0}$, and $b$ must be distinct vertices in $V_{v-w}$. But $\left|V_{v-w}\right|=2$, so this cannot happen. If $\left(0_{0}, 1_{0}\right)$ is an edge in the 4 -cycle of some $H$, then there must be an edge in the 4-cycle of the form $\left(a_{1}, b_{1}\right)$, a contradiction to the restricted covering. So, $v-w>2$ is necessary.

Similar to the argument in Theorem 2, an $H$-covering of $K(v, w)$ with padding $P$ where $|E(P)|=-\mid E(K(v, w) \mid(\bmod 5)$ would be minimal.

Case 1. Suppose $v \equiv 0(\bmod 5)$ and $w \equiv 2(\bmod 5)$. First, $K(5,2)$ can be covered with $\left\{\left[0_{0}, 0_{1}, 2_{0}, 1_{0} ; 1_{1}\right],\left[2_{0}, 0_{1}, 1_{0}, 1_{1}\right.\right.$; $\left.\left.0_{0}\right]\right\}$, and this has a padding $P$ with $E(P)=\left\{\left(2_{0}, 0_{1}\right)\right\}$ and so $|E(P)|=1$. For general $v$ and $w, K(v, w)=K(5,2) \cup K_{v-w-3,3} \cup$ 
$K_{v-w, w-2}$, where the vertex set of $K(5,2)$ is $\left\{0_{0}, 1_{0}, 2_{0}, 0_{1}, 1_{1}\right\}$ and the hole is on vertex set $\left\{0_{1}, 1_{1}\right\}$, the partite sets of $K_{v-w-3,3}$ are $\left\{3_{0}, 4_{0}, \ldots,(v-w-1)_{0}\right\}$ and $\left\{0_{0}, 1_{0}, 2_{0}\right\}$, and the partite sets of $K_{v-w, w-2}$ are $V_{v-w}$ and $\left\{2_{1}, 3_{1}, \ldots,(w-1)_{1}\right\}$. Now, $K_{v-w-3,3}$ and $K_{v-w, w-2}$ can be decomposed [13]. Taking these decompositions along with the above covering of $K(5,2)$ yields a covering of $K(v, w)$ with padding $P$, where $E(P)=$ $\left\{\left(2_{0}, 0_{1}\right)\right\}$, and so $|E(P)|=1=-|E(K(v, w))|(\bmod 5)$.

Case 2. Suppose $v \equiv 0(\bmod 5)$ and $w \equiv 3(\bmod 5)$ or $v \equiv 3$ $(\bmod 5)$, and $w \equiv 4(\bmod 5)$. Consider $K(v, w)=K_{v-w} U$ $K_{v-w, w}$, where $V\left(K_{v-w}\right)=V_{v-w}$ and the partite sets of $K_{v-w, w}$ are $V_{v-w}$ and $V_{w}$ and $v-w \equiv 2(\bmod 5)$ and $w \equiv 3(\bmod 5)$ or $v-w \equiv 4(\bmod 5)$, and $w \equiv 4(\bmod 5)$. There is a maximal packing of $K_{v-w}$ where $v-w \equiv 2(\bmod 5)$ or $v-w \equiv 4(\bmod 5)$ with $\left|E\left(L_{1}\right)\right|=1$ by Theorem 1 . There is a maximal packing of $K_{v-w, w}$ with $\left|E\left(L_{2}\right)\right|=1$ by Theorem 3 . Therefore, there is a minimal covering of $K(v, w)$ with padding $P$, where $|E(P)|=$ $3=-|E(K(v, w))|(\bmod 5)$.

Case 3. Suppose $v \equiv 0(\bmod 5)$, and $w \equiv 4(\bmod 5)$. Consider $K(v, w)=K_{v-w} \cup K_{v-w, w}$, as in Case 2, where $v-w \equiv 1$ $(\bmod 5)$, and $w \equiv 4(\bmod 5)$. There is a minimal covering of $K_{v-w, w}$ with padding $P$, where $|E(P)|=1$ by Theorem 4 and $K_{v-w}$, where $v-w \equiv 1(\bmod 5)$ is decomposable [9]. Therefore, there is a minimal covering of $K(v, w)$ with padding $P$, where $|E(P)|=1=-|E(K(v, w))|(\bmod 5)$.

Case 4. Suppose $v \equiv 1(\bmod 5)$ and $w \equiv 2(\bmod 5)$. Consider $K(v, w)=K_{v-w} \cup K_{v-w, w}$, as in Case 2, where $v-w \equiv 4(\bmod 5)$ and $w \equiv 2(\bmod 5)$. There is a maximal packing of $K_{v-w}$ with leave $L_{1}$, where $\left|E\left(L_{1}\right)\right|=1$ by Theorem 1 and, without loss of generality, $E\left(L_{1}\right)=\left\{\left(0_{0}, 2_{0}\right)\right\}$. There is a maximal packing of $K_{v-w, w}$ with leave $L_{2}$, where $\left|E\left(L_{2}\right)\right|=3$ and $E\left(L_{2}\right)=$ $\left\{\left(2_{0}, 0_{1}\right),\left(0_{1}, 3_{0}\right),\left(3_{0}, 1_{1}\right)\right\}$ by Theorem 3 . These two packings combined with $\left\{\left[2_{0}, 0_{1}, 3_{0}, 1_{1} ; 0_{0}\right]\right\}$ yield a covering of $K(v, w)$ with padding $P$, where $E(P)=\left\{\left(2_{0}, 1_{1}\right)\right\}$, so $|E(P)|=1=$ $-|E(K(v, w))|(\bmod 5)$.

Case 5. Suppose $v \equiv 1(\bmod 5)$ and $w \equiv 4(\bmod 5)$. Consider $K(v, w)=K_{v-w} \cup K_{v-w, w}$, as in Case 2 , where $v-w \equiv 2(\bmod 5)$ and $w \equiv 4(\bmod 5)$. There is a maximal packing of $K_{v-w}$ with leave $L_{1}$, where $\left|E\left(L_{1}\right)\right|=1$ by Theorem 1 and, without loss of generality, $E\left(L_{1}\right)=\left\{\left(0_{1}, 2_{1}\right)\right\}$. There is a maximal packing of $K_{v-w, w}$ with leave $L_{2}$, where $\left|E\left(L_{2}\right)\right|=3$ and $E\left(L_{2}\right)=$ $\left\{\left(2_{0}, 0_{1}\right),\left(0_{1}, 3_{0}\right),\left(3_{0}, 1_{1}\right)\right\}$ by Theorem 3 . These two packings combined with $\left\{\left[0_{1}, 3_{0}, 1_{1}, 2_{0} ; 2_{1}\right]\right\}$ yield a covering of $K(v, w)$ with padding $P$, where $E(P)=\left\{\left(2_{0}, 1_{1}\right)\right\}$, so $|E(P)|=1=$ $-|E(K(\nu, w))|(\bmod 5)$.

Case 6. Suppose $v \equiv 1(\bmod 5)$ and $w \equiv 3(\bmod 5)$. Consider $K(v, w)=K_{v-w+1} \cup K_{v-w, w-1}$, where $V\left(K_{v-w+1}\right)=V_{v-w} \cup\left\{w_{1}\right\}$, and the partite sets of $K_{v-w, w-1}$ are $V_{v-w} \cup\left\{w_{1}\right\}$ and $V_{w} \backslash\left\{w_{1}\right\}$. Then there is a maximal packing of $K_{v-w+1}$ with leave $L_{1}$, where $\left|E\left(L_{1}\right)\right|=1$ by Theorem 1 , and there is a maximal packing of $K_{v-w, w-1}$ with leave $L_{2}$, where $\left|E\left(L_{2}\right)\right|=1$ by Theorem 3. Therefore, we can add an additional copy of $H$ which includes the edges in $L_{1}$ and $L_{2}$. So, there is a minimal covering of $K(v, w)$ with padding $P$, where $|E(P)|=3=$ $-|E(K(v, w))|(\bmod 5)$.

Case 7. Suppose $v \equiv 2(\bmod 5)$ and $w \equiv 0(\bmod 5)$, or $v \equiv$ $4(\bmod 5)$ and $w \equiv 0(\bmod 5)$. Consider $K(v, w)=K_{v-w} \cup$ $K_{v-w, w}$, as in Case 2 , where $v-w \equiv 2(\bmod 5)$, and $w \equiv 0$ $(\bmod 5)$ or $v-w \equiv 4(\bmod 5)$, and $w \equiv 0(\bmod 5)$. There is a minimal covering of $K_{v-w}$ with padding $P$, where $|E(P)|=4$ by Theorem 2, and $K_{v-w, w}$ is decomposable [13]. Therefore, there is a minimal covering of $K(v, w)$ with padding $P$ where $|E(P)|=4=-|E(K(v, w))|(\bmod 5)$.

Case 8. Suppose $v \equiv 2(\bmod 5)$ and $w \equiv 1(\bmod 5)$. Consider $K(v, w)=K_{v-w} \cup K_{v-w, w}$, as in Case 2, where $v-w \equiv 1$ $(\bmod 5)$ and $w \equiv 1(\bmod 5)$. There is a minimal covering of $K_{v-w, w}$ with padding $P$, where $|E(P)|=4$ by Theorem 4 and $K_{v-w}$, where $v-w \equiv 1(\bmod 5)$ is decomposable [9]. Therefore, there is a minimal covering of $K(v, w)$ with padding $P$, where $|E(P)|=4=-|E(K(v, w))|(\bmod 5)$.

Case 9. Suppose $v \equiv 2(\bmod 5)$ and $w \equiv 3(\bmod 5)$. Consider $K(v, w)=K_{v-w} \cup K_{v-w, w}$, as in Case 2, where $v-w \equiv 4$ $(\bmod 5)$ and $w \equiv 3(\bmod 5)$. There is a maximal packing of $K_{v-w}$ with leave $L_{1}$, where $\left|E\left(L_{1}\right)\right|=1$ by Theorem 1 and, without loss of generality, $E\left(L_{1}\right)=\left\{\left(0_{0}, 1_{0}\right)\right\}$. There is a maximal packing of $K_{v-w, w}$ with leave $L_{2}$, where $\left|E\left(L_{2}\right)\right|=$ 2 and $E\left(L_{2}\right)=\left\{\left(0_{0}, 3_{1}\right),\left(2_{0}, 0_{1}\right)\right\}$ by Theorem 3 . These two packings combined with $\left\{\left[0_{0}, 0_{1}, 2_{0}, 3_{1} ; 1_{0}\right]\right\}$ yield a covering of $K(v, w)$ with padding $P$, where $E(P)=\left\{\left(0_{0}, 0_{1}\right),\left(2_{0}, 3_{1}\right)\right\}$, so $|E(P)|=2=-|E(K(v, w))|(\bmod 5)$.

Case 10. Suppose $v \equiv 3(\bmod 5)$ and $w \equiv 1(\bmod 5)$. Consider $K(v, w)=K_{v-w} \cup K_{v-w, w}$, as in Case 2, where $v-w \equiv 2$ $(\bmod 5)$ and $w \equiv 1(\bmod 5)$. There is a maximal packing of $K_{v-w}$ with leave $L_{1}$, where $\left|E\left(L_{1}\right)\right|=1$ by Theorem 1 and, without loss of generality, $E\left(L_{1}\right)=\left\{\left(1_{0}, 2_{0}\right)\right\}$. These is a maximal packing of $K_{v-w, w}$ with leave $L_{2}$, where $\left|E\left(L_{2}\right)\right|=$ 2 and $E\left(L_{2}\right)=\left\{\left(2_{0}, 1_{1}\right),\left(3_{0}, 0_{1}\right)\right\}$ by Theorem 3 . There two packings combined with $\left\{\left[2_{0}, 0_{1}, 3_{0}, 1_{1} ; 1_{0}\right]\right\}$ yield a covering of $K(v, w)$ with padding $P$, where $E(P)=\left\{\left(2_{0}, 0_{1}\right),\left(3_{0}, 1_{1}\right)\right\}$, so $|E(P)|=2=-|E(K(v, w))|(\bmod 5)$.

Case 11. Suppose $v \equiv 3(\bmod 5)$ and $w \equiv 0(\bmod 5)$. Consider $K(v, w)=K_{v-w} \cup K_{v-w, w}$, as in Case 2 , where $v-w \equiv 3(\bmod 5)$ and $w \equiv 0(\bmod 5)$. There is a minimal covering of $K_{v-w}$ with padding $P$, where $|E(P)|=2$ by Theorem 2 , and $K_{v-w, w}$ is decomposable [13]. Therefore, there is a minimal covering of $K(v, w)$ with padding $P$, where $|E(P)|=2=-|E(K(v, w))|$ $(\bmod 5)$.

Case 12. Suppose $v \equiv 3(\bmod 5)$ and $w \equiv 2(\bmod 5)$. Consider $K(v, w)=K_{v-w} \cup K_{v-w, w}$, as in Case 2, where $v-w \equiv 1$ $(\bmod 5)$ and $w \equiv 2(\bmod 5)$. There is a minimal covering of $K_{v-w, w}$ with padding $P$, where $|E(P)|=3$ by Theorem 4 and $K_{v-w}$, where $v-w \equiv 1(\bmod 5)$ is decomposable [9]. Therefore, there is a minimal covering of $K(v, w)$ with padding $P$, where $|E(P)|=3=-|E(K(v, w))|(\bmod 5)$.

Case 13. Suppose $v \equiv 4(\bmod 5)$ and $w \equiv 1(\bmod 5)$. As in Case 6, we have $K(v, w)=K_{v-w+1} \cup K_{v-w, w-1}$. Then there is 
a minimal covering of $K_{v-w+1}$ with padding $P$, where $|E(P)|=$ 4 by Theorem 2 and $K_{v-w, w-1}$ is decomposable [13]. Therefore, there is a minimal covering of $K(v, w)$ with padding $P$, where $|E(P)|=4=-|E(K(v, w))|(\bmod 5)$.

Case 14. Suppose $v \equiv 4(\bmod 5)$ and $w \equiv 3(\bmod 5)$. Consider $K(v, w)=K_{v-w} \cup K_{v-w, w}$, as in Case 2, where $v-w \equiv 1$ $(\bmod 5)$ and $w \equiv 3(\bmod 5)$. There is a minimal covering of $K_{v-w, w}$ with padding $P$, where $|E(P)|=2$ by Theorem 4 and $K_{v-w}$, where $v-w \equiv 1(\bmod 5)$ is decomposable [9]. Therefore, there is a minimal covering of $K(v, w)$ with padding $P$, where $|E(P)|=2=-|E(K(v, w))|(\bmod 5)$.

Theorem 8. A minimal unrestricted $H$-covering of $K(v, w)$ has padding $P$ where

(1) when $v-w>2,|E(P)|=-|E(K(v, w))|(\bmod 5)$,

(2) when $v-w=1,|E(P)|=(2 / 3) w$ for $w \equiv 0(\bmod 3)$, $|E(P)|=2(w+5) / 3$ for $w \equiv 1(\bmod 3),|E(P)|=(2 w+$ $5) / 3$ for $w \equiv 2(\bmod 3)$,

(3) when $v-w=2,|E(P)|=5-\ell$, where $\ell=$ $\mid E(K(v, w) \mid(\bmod 5)$ for $v \neq 6$ and $|E(P)|=6$ for $v=6$.

Proof. When $v-w>2$, the necessary and sufficient conditions follow from Theorem 7 . When $v-w=1, K(v, w) \cong$ $K_{1, w}$ and the necessary and sufficient conditions follow from Theorem 5.

When $v-w=2$, similar to the argument in Theorem 2, an $H$-covering of $K(v, w)$ with padding $P$ must satisfy $|E(P)| \equiv$ $-\mid E(K(\nu, w) \mid(\bmod 5)$. Since an $H$-decomposition of $K(v, w)$ does not exist for $w \equiv 2(\bmod 5)[13]$, the necessary conditions follow for $v-w=2$ and $v \neq 6$. For $v=6$, since $\mid E(K(6$, $4)) \mid=9$, then an unrestricted $H$-covering of $K(6,4)$ with padding $P$ where $|E(P)|=1$ would be minimal. However, in such a covering, there are only two copies of $H$. Edge $\left(0_{0}, 1_{0}\right)$ cannot be the pendant edge of a copy of $H$ in such a covering since this copy would have 2 edges in the padding. If edge $\left(0_{0}, 1_{0}\right)$ is in a copy of $H$ and is not the pendant edge, then this copy of $H$ must be of the form $\left[0_{0}, 1_{0}, a_{1}, b_{1} ; c_{1}\right]$ for some distinct $a_{1}, b_{1}, c_{1} \in\left\{0_{1}, 1_{1}, 2_{1}, 3_{1}\right\}$. However, the complement of this graph in $K(6,4)$ is not a copy of $H$. Therefore, no such $H$-covering of $K(6,4)$ exists, and a minimal unrestricted $H$-covering of $K(6,4)$ with padding $P$, where $|E(P)|=6$ would be minimal. The set $\left\{\left[1_{0}, 1_{1}, 0_{1}, 0_{0} ; 2_{1}\right],\left[0_{0}, 2_{1}, 1_{0}, 3_{1} ; 1_{1}\right],\left[1_{0}, 3_{1}, 0_{0}, 2_{1} ; 0_{1}\right]\right\}$ is an unrestricted $H$-covering of $K(6,4)$ with padding $P$ where $E(P)=\left\{\left(0_{1}, 1_{1}\right),\left(1_{0}, 2_{1}\right),\left(0_{0}, 2_{1}\right),\left(1_{0}, 2_{1}\right),\left(1_{0}, 3_{1}\right),\left(0_{0}, 3_{1}\right)\right\}$. So $|E(P)|=6$, and the covering is minimal.

Case 1. Suppose $v-w=2$ and $w \equiv 0(\bmod 5) ; w \geq 5$. Then $K(v, w)=K(7,5) \cup K_{2, w-5}$ where the vertex set of $K(7,5)$ is $\left\{0_{0}, 1_{0}, 0_{1}, 1_{1}, 2_{1}, 3_{1}, 4_{1}\right\}$ and the hole is on vertex set $\left\{0_{1}, 1_{1}, 2_{1}, 3_{1}, 4_{1}\right\}$, and the partite sets of $K_{2, w-5}$ are $\left\{0_{0}, 1_{0}\right\}$ and $\left\{5_{1}, 6_{1}, \ldots,(w-1)_{1}\right\}$. There is an $H$ decomposition of $K_{2, w-5}$ [13], and the set $\left\{\left[1_{0}, 1_{1}, 0_{1}, 0_{0} ; 2_{1}\right]\right.$, $\left.\left[0_{0}, 3_{1}, 1_{0}, 4_{1} ; 2_{1}\right],\left[1_{1}, 0_{1}, 1_{0}, 2_{1} ; 0_{0}\right]\right\}$ is an unrestricted $H$ covering of $K(7,5)$ with padding $P$, where $E(P)=$ $\left\{\left(0_{1}, 1_{1}\right),\left(0_{1}, 1_{1}\right),\left(1_{1}, 2_{1}\right),\left(1_{0}, 2_{1}\right)\right\}$ and $|E(P)|=4$. So, there is an unrestricted covering of $K(v, w)$ with padding $P$, where $|E(P)|=4=-|E(K(\nu, w))|(\bmod 5)$.

Case 2. Suppose $v-w=2, w \equiv 1(\bmod 5) ; w \geq 6$. Then, as in Case $1, K(v, w)=K(8,2) \cup K_{2, w-6}$. There is an $H$-decomposition of $K_{2, w-6}[13]$, and the set $\left\{\left[1_{0}, 1_{1}, 0_{1}\right.\right.$, $\left.\left.0_{0} ; 2_{1}\right],\left[1_{0}, 3_{1}, 0_{0}, 4_{1} ; 0_{1}\right],\left[0_{1}, 2_{1}, 1_{0}, 5_{1} ; 1_{1}\right]\right\}$ is an unrestricted $H$-covering of $K(8,6)$ with padding $P$, where $E(P)=$ $\left\{\left(0_{1}, 1_{1}\right),\left(1_{0}, 2_{1}\right)\right\}$ and $|E(P)|=2$. So, there is an unrestricted covering of $K(v, w)$ with padding $P$, where $|E(P)|=2=$ $-|E(K(v, w))|(\bmod 5)$.

Case 3. Suppose $v-w=2$ and $w \equiv 2(\bmod 5) ; w \geq 7$. Then, as in Case $1, K(v, w)=K(9,7) \cup K_{2, w-7}$. There is an $H$ decomposition of $K_{2, w-7}$ [13], and the set $\left\{\left[1_{0}, 1_{1}, 0_{1}, 0_{0} ; 2_{1}\right]\right.$, $\left.\left[1_{0}, 3_{1}, 0_{0}, 4_{1} ; 0_{1}\right],\left[0_{1}, 2_{1}, 1_{0}, 5_{1} ; 1_{1}\right],\left[0_{0}, 5_{1}, 1_{0}, 6_{1} ; 0_{1}\right]\right\}$ is an unrestricted $H$-covering of $K(9,7)$ with padding $P$, where $E(P)=\left\{\left(0_{1}, 1_{1}\right),\left(1_{0}, 2_{1}\right),\left(0_{0}, 0_{1}\right),\left(0_{0}, 5_{1}\right),\left(1_{0}, 5_{1}\right)\right\}$ and $|E(P)|=5$. So, there is an unrestricted covering of $K(v, w)$ with padding $P$, where $|E(P)|=5=5-\ell$, where $\ell=0=$ $-|E(K(v, w))|(\bmod 5)$.

Case 4. Suppose $v-w=2$ and $w \equiv 3(\bmod 5)$. Then, as in Case $1, K(v, w)=K(5,3) \cup K_{2, w-3}$. There is an $H$-decomposition of $K_{2, w-3}[13]$, and the set $\left\{\left[1_{0}, 0_{0}, 0_{1}, 1_{1} ; 2_{1}\right],\left[0_{1}, 2_{1}, 0_{0}, 1_{1} ; 1_{0}\right]\right\}$ is an unrestricted $H$-covering of $K(5,3)$ with padding $P$, where $E(P)=\left\{\left(0_{1}, 1_{1}\right),\left(0_{1}, 1_{1}\right),\left(0_{1}, 2_{1}\right)\right\}$ and $|E(P)|=3$. So, there is an unrestricted covering of $K(v, w)$ with padding $P n$ where $|E(P)|=3=-|E(K(v, w))|(\bmod 5)$.

Case 5. Suppose $v-w=2$ and $w \equiv 4(\bmod 5) ; w \geq 9$. Then, as in Case $1, K(v, w)=K(11,9) \cup K_{2, w-9}$. There is an $H$ decomposition of $K_{2, w-9}$ [13], and the set $\left\{\left[1_{0}, 1_{1}, 0_{1}, 0_{0} ; 2_{1}\right]\right.$, $\left.\left[0_{0}, 7_{1}, 1_{0}, 8_{1} ; 1_{1}\right],\left[0,0_{0}, 1_{0}, 6_{1} ; 2_{1}\right],\left[0_{0}, 3_{1}, 1_{0}, 4_{1} ; 0_{1}\right]\right\}$ is an unrestricted covering of $K(11,9)$ with padding $P$, where $E(P)=\left\{\left(0_{1}, 1_{1}\right)\right\}$ and $|E(P)|=1$. So, there is an unrestricted covering of $K(v, w)$ with padding $P$, where $|E(P)|=1=$ $-|E(K(v, w))|(\bmod 5)$.

\section{Conclusion}

Motivated by experimental designs and comparisons of samples, we have given necessary and sufficient conditions for the $H$-packings and $H$-coverings of complete graphs, complete bipartite graphs, and complete graphs with a hole, where $H$ is a 4 -cycle with a pendant edge. For complete bipartite graphs and complete graphs with a hole, we have given both restricted and unrestricted coverings.

\section{References}

[1] T. Kirkman, "On a problem in combinatorics," Cambridge and Dublin Mathematics Journal, vol. 2, pp. 191-204, 1847.

[2] J. Schönheim, “On maximal systems of K-tuples," Studia Scientiarum Mathematicarum Hungarica, vol. 1, pp. 363-368, 1966.

[3] J. Schönheim and A. Bialostocki, "Packing and covering of the complete graph with 4-cycles," Canadian Mathematical Bulletin, vol. 18, no. 5, pp. 703-708, 1975. 
[4] A. E. Brouwer, "Optimal packings of $K_{4}$ 's into a $K_{n}$ ", Journal of Combinatorial Theory A, vol. 26, no. 3, pp. 278-297, 1979.

[5] J. A. Kennedy, "Maximum packings of $K_{n}$ with hexagons," The Australasian Journal of Combinatorics, vol. 7, pp. 101-110, 1993.

[6] J. A. Kennedy, "Maximum packings of $K_{n}$ with hexagons: corrigendum," The Australasian Journal of Combinatorics, vol. 10, p. 293, 1994.

[7] M. K. Fort Jr. and G. A. Hedlund, "Minimal coverings of pairs by triples," Pacific Journal of Mathematics, vol. 8, pp. 709-719, 1958.

[8] J. A. Kennedy, "Minimum coverings of $K_{n}$ with hexagons," The Australasian Journal of Combinatorics, vol. 16, pp. 295-303, 1997.

[9] J.-C. Bermond, C. Huang, A. Rosa, and D. Sotteau, "Decomposition of complete graphs into isomorphic subgraphs with five vertices," Ars Combinatoria, vol. 10, pp. 211-254, 1980.

[10] J.-C. Bermond and J. Schönheim, "G-decomposition of $K_{n}$, where G has four vertices or less," Discrete Mathematics, vol. 19, no. 2, pp. 113-120, 1977.

[11] Q.-D. Kang, L.-D. Yuan, and S.-X. Liu, "Graph designs for all graphs with six vertices and eight edges," Acta Mathematicae Applicatae Sinica, vol. 21, no. 3, pp. 469-484, 2005.

[12] Q. Kang, H. Zhao, and C. Ma, "Graph designs for nine graphs with six vertices and nine edges," Ars Combinatoria, vol. 88, pp. 379-395, 2008.

[13] B. Coker, G. Coker, and R. Gardner, "Decompositions of various complete graphs into isomorphic copies of the 4-cycle with a pendant edge," International Journal of Pure and Applied Mathematics, vol. 74, no. 4, pp. 485-492, 2012.

[14] D. E. Bryant, D. G. Hoffman, and C. A. Rodger, "5-cycle systems with holes," Designs, Codes and Cryptography, vol. 8, no. 1-2, pp. 103-108, 1996, Special issue dedicated to Hanfried Lenz.

[15] D. E. Bryant, C. A. Rodger, and E. R. Spicer, "Embeddings of $m$-cycle systems and incomplete $m$-cycle systems: $m \leq 14$," Discrete Mathematics, vol. 171, no. 1-3, pp. 55-75, 1997.

[16] E. Mendelsohn and A. Rosa, "Embedding maximal packings of triples," Congressus Numerantium, vol. 40, pp. 235-247, 1983.

[17] R. Gardner and W. Surber, "Restricted and unrestricted hexagon coverings of the complete bipartite graph". 


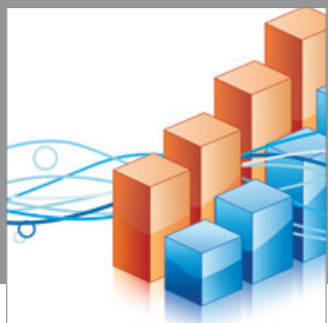

Advances in

Operations Research

mansans

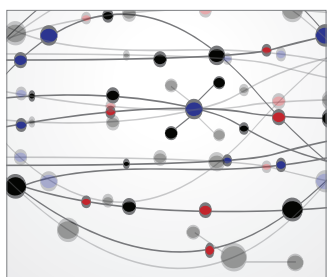

The Scientific World Journal
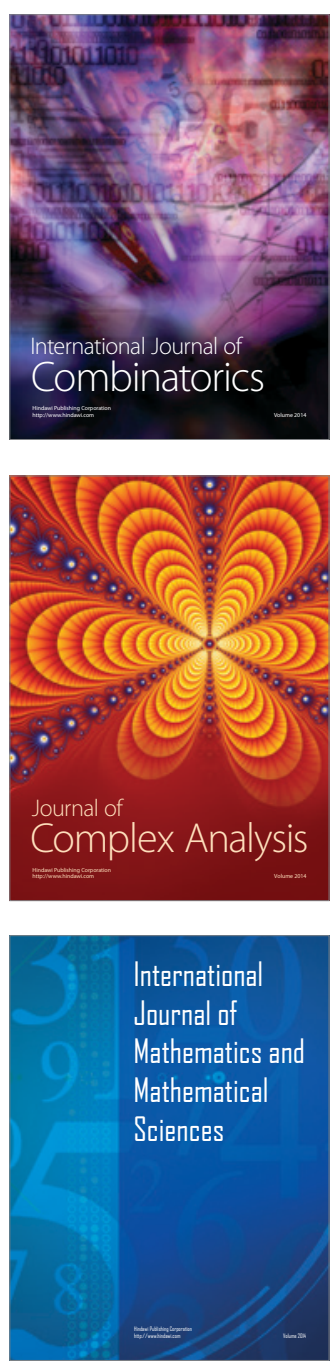
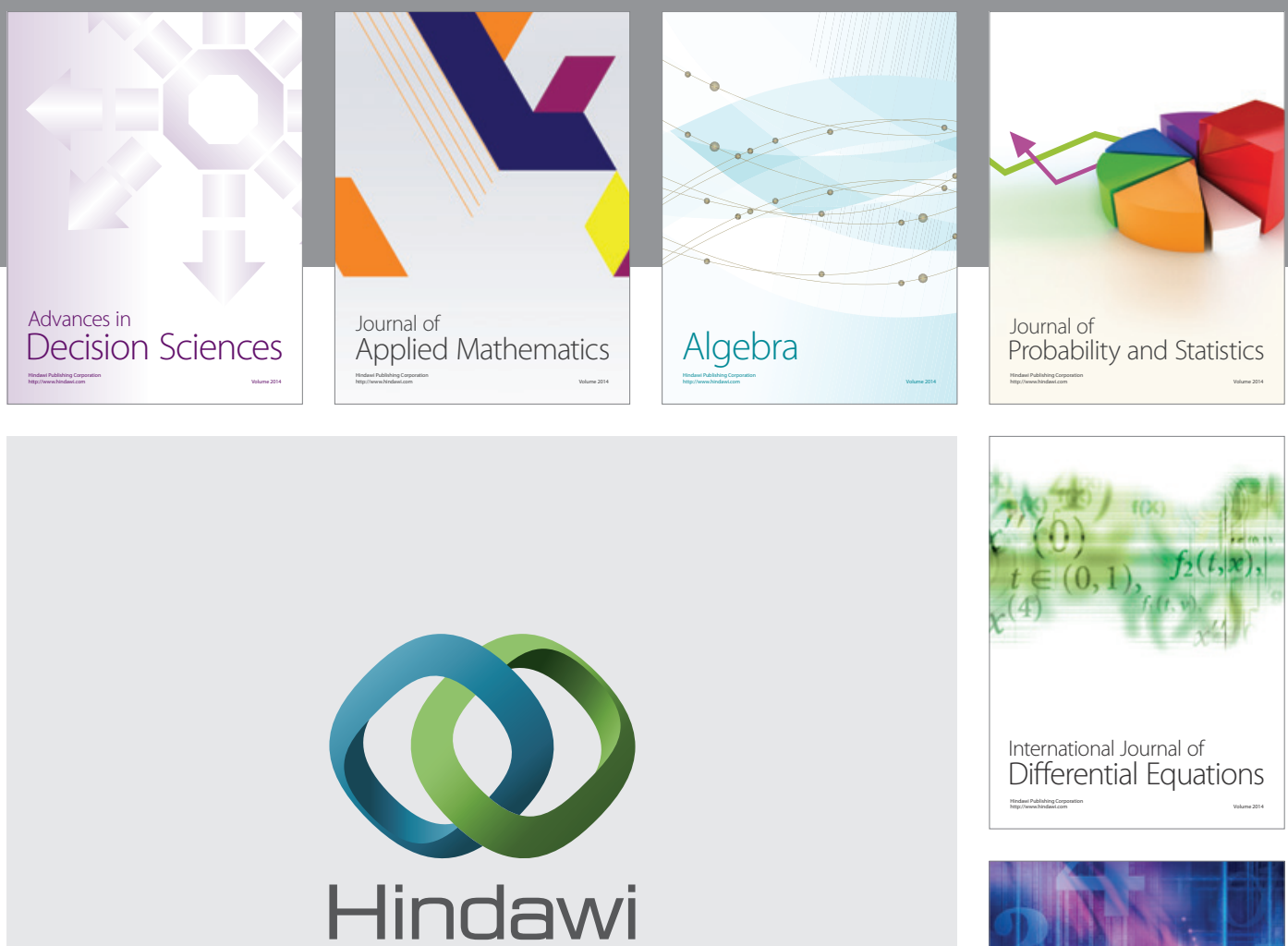

Submit your manuscripts at http://www.hindawi.com
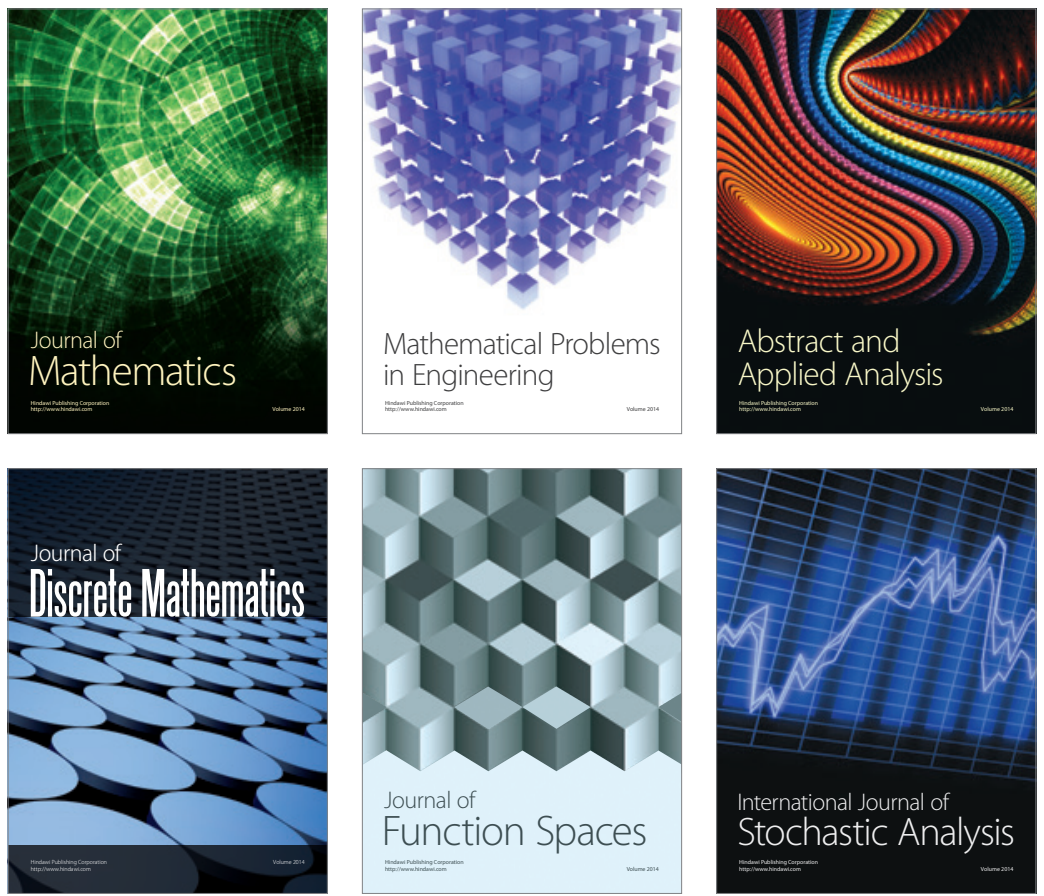

Journal of

Function Spaces

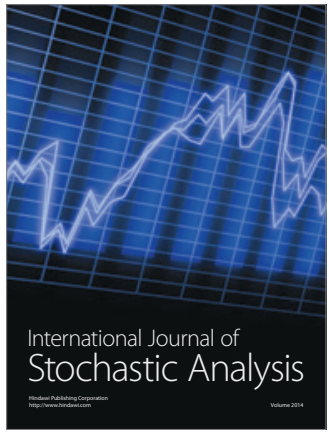

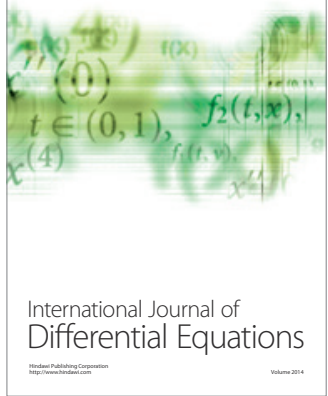
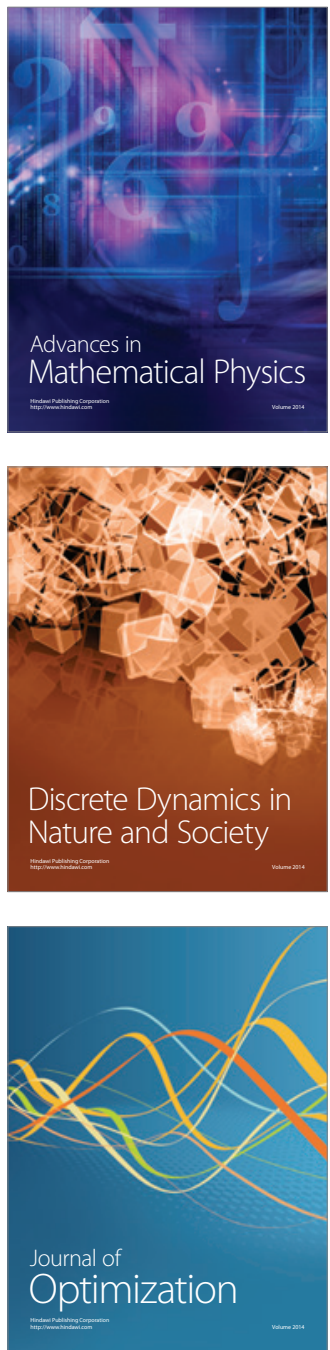